\title{
Selección de campos para la implementación de solar EOR como proceso térmico de recobro mejorado en Colombia
}

\author{
Martha Patricia Medina Casas ${ }^{1 *}$; Angélica María Gutiérrez Ramírez ${ }^{1}$; Maria Patricia Amorin Figueroa ${ }^{2}$ Freddy Humberto \\ Escobar Macualo ${ }^{1}$; Camilo Andrés Guerrero Martin ${ }^{3}$ \\ ${ }^{1}$ Universidad Surcolombiana (USCO). Avenida Pastrana Borrero - Carrera 1. Neiva, Colombia. \\ ${ }^{2}$ Universidad de Oriente. Via Alterna, Barcelona 6001, Anzoátegui, Venezuela \\ ${ }^{3}$ Universidade Federal do Rio de Janeiro (UFRJ). Av. Pedro Calmon. n 550 - Prédio da Reitoria, $2^{\circ}$ andar Cidade Universitária. \\ Rio de Janeiro, Brasil.
}

Email: *u20152143512@usco.edu.co

\begin{abstract}
Resumen
La tecnología Solar EOR actualmente desempeña un papel importante en la combinación de tecnologías EOR para procesos de recobro térmico con inyección continua de vapor; dado que, en lugar de quemar gas natural para producir vapor, Solar EOR involucra el uso de la tecnología de concentración de energía solar $(C S P)$ para producir vapor. El mecanismo de operación consiste en instalar espejos parabólicos protegidos por un invernadero, que reflejan y concentran la luz solar en los receptores que recogen energía solar y luego la convierten en calor. El calor se usa para producir vapor del agua; Solar EOR puede generar la misma calidad y temperatura de vapor como gas natural. En Colombia existen zonas de interés en donde se encuentran campos petrolíferos que cumplen los criterios de las propiedades petrofísicas de fluidos y del yacimiento, así como la irradiación normal directa (DNI) necesaria para la instalación de esta tecnología, por lo tanto, el uso de Solar EOR podría reducir la demanda que se requiere de gas natural para $E O R$, que puede redirigirse a otras actividades económicas tales como generación de energía, desalinización de agua y como materia prima y energía para procesos industriales. El presente trabajo busca seleccionar los campos candidatos para la aplicación de esta tecnología.
\end{abstract}

Palabras clave: recobro térmico, inyección continua de vapor, irradiación normal directa (DNI), concentración de energía solar, generación de energía, gas natural.

\section{Selection of Fields for the Implementation of Solar EOR as a Thermal Process of Improved Recovery in Colombia}

\begin{abstract}
Solar EOR technology currently plays an important role in the combination of EOR technologies for thermal recovery processes with continuous steam injection; since, instead of burning natural gas to produce steam, Solar EOR involves the use of solar energy concentration technology (CSP) to produce steam. The mechanism of operation consists in installing parabolic mirrors protected by a greenhouse, which reflect and concentrate the sunlight in the receivers that collect solar energy and then turn it into heat. The heat is used to produce steam from the water; Solar EOR can generate the same quality and temperature of steam as natural gas. In Colombia there are areas of interest where oil fields meet the criteria of the petrophysical properties of fluids and the deposit, as well as the direct normal irradiation (DNI) necessary for the installation of this technology, therefore, the use of Solar EOR could reduce the demand that natural gas is required for EOR, which can be redirected to other economic activities such as power generation, water desalination and as raw material and energy for industrial processes. This paper seeks to select the candidate fields for the application of this technology.
\end{abstract}

Keywords: thermal recovery, continuous steam injection, direct normal irradiation (DNI), concentration of solar energy, power generation, natural gas.

Cita: Medina Casas, M. P., Gutiérrez Ramírez, A. M., Amorin Figueroa, M. P., Escobar Macualo, F. H. y Guerrero Martin, C. A. (2019). Selección de campos para la implementación de solar EOR como proceso técnivo de recobro mejorado. Revista Fuentes: El reventón energético, $17(2), 27-37$. 


\section{Introducción}

La alta demanda energética a la que se enfrenta la industria de los hidrocarburos es cada vez mayor, a la fecha el consumo mundial es de 89 millones de barriles diarios, algo más de 14 millones de metros cúbicos, los hidrocarburos al ser un recurso natural no renovable, está en constante búsqueda de la reinvención y de la maximización de producción de los campos petrolíferos, es decir, incrementar el factor de recuperación de crudos. En la actualidad, los crudos de naturaleza pesados y extra - pesados representan el $70 \%$ del petróleo restante en el mundo, por ende, son una cifra significativa de alto interés para ser explotados, estos petróleos no pueden fluir a menos que se los caliente y se reduzca su viscosidad lo suficiente para permitirles fluir hacia los pozos productores, es aquí donde los métodos de recobro mejorado garantizan la producción de los mismos implementando recobro térmico.

Durante la recuperación térmica, el petróleo crudo sufre cambios físicos y químicos debido a los efectos del calor suministrado. Las propiedades físicas tales como la viscosidad, la gravedad específica y la tensión interfacial son alteradas. Los cambios químicos involucran diferentes reacciones tales como el craqueo, que es la destrucción de los enlaces carbono-carbono para generar compuestos de peso molecular más bajo y la deshidrogenación, la cual es la ruptura de enlaces carbono-hidrógeno (Schlumberger, 2018).

La producción de crudo en Colombia es en su mayoría es de tipo pesado y extrapesado, su tendencia tiende a incrementar con el paso del tiempo, dado que mientras en el año 2000 únicamente el $10 \%$ del petróleo producido en el país correspondía a crudo pesado, en la actualidad dicho porcentaje supera el 50\% (Malagón, 2016), por consiguiente, la implementación de técnicas para la producción de estos hidrocarburos se hace cada vez más necesaria.

En muchos proyectos, los costos asociados con la generación de vapor impiden su implementación en campo por lo que adicionalmente, se presenta una visualización de oportunidades para Colombia en cuanto al uso de energías limpias para la recuperación de hidrocarburos pesados utilizando fuentes alternativas como la energía solar (Pérez R., et al. 2018). Solar EOR propone producir energía utilizando la radiación solar para redirigir esta energía hasta finalmente utilizarla en convertir el agua en vapor y utilizarlos como un método de recobro térmico en campos petrolíferos que cumplan los criterios de implantación de esta técnica. Desde el punto de vista académico científico este proyecto se justifica considerando que se plantean como resultados la factibilidad de la implementación de una planta de energía solar para procesos térmicos de recobro mejorado en Colombia, guiados por el estudio de los campos petroleros sobre este territorio que se caractericen por ser los prototipos indicados para la implementación de dicha técnica, esto es, que cumplan con las propiedades tanto de la roca, de los fluidos y de la interacción roca-fluidos.

\section{Fundamentación conceptual}

La industria energética proveniente de los hidrocarburos se enfrenta cada día a la necesidad de expandir el rango de operación de proyectos de recobro mejorado haciendo que las tecnologías emergentes de recobro térmico se presenten con un potencial de mejoramiento de la eficiencia de recobro de los campos petroleros, ya que ofrecen posibles soluciones tecnológicas al desarrollo convencional de métodos de recobro mejorado.

En Colombia el volumen de petróleo estimado en el subsuelo (Petróleo Original En Sitio - POES) es de 52 mil millones de barriles, y el petróleo recuperado a la fecha es del $16 \%$ y el factor de recobro promedio con desarrollos actuales es de $24 \%$ frente al $35 \%$ promedio de otros países, con una meta de incrementar inicialmente a $26 \%$ y luego al $30 \%$, con este recobro se podría hacer la reposición de 5000 Millones de barriles de reservas en el mediano plazo (Pardo, D. F. 2015).

Con el fin de poder cumplir con las metas establecidas respecto al factor de recobro la industria de los hidrocarburos debe priorizar las tecnologías emergentes dependiendo de su potencial de aplicación y teniendo en cuenta las necesidades de los campos de interés; por lo anterior, se propone una metodología que permite identificar los parámetros esenciales para aplicar la tecnología de solar EOR, con base a criterios clave para implementar una tecnología que busca reemplazar el método de recobro de inyección de vapor convencional por uno que utiliza una fuente de energía renovable como lo es la energía solar para convertir el agua en vapor en lugar de depender del gas natural como combustible para dicho proceso, esta tecnología es denominada Solar EOR; de esta forma enfocar Solar EOR en los Campos petroleros que tienen mayor aplicabilidad.

Las fuentes de energía renovable son un desafío para el desarrollo tecnológico e ingenieril de los científicos alrededor del mundo, como plan de contingencia a 
los problemas generados por el cambio climático producto de la contaminación y deterioro del medio ambiente. En Colombia, el desarrollo de tecnologías que involucran fuentes de energía renovable ha sido lento, a pesar de ser un país que posee matriz energética tanto en combustibles fósiles como en recursos renovables (Vidal E., \& Fontalvo C., 2018).

En el caso de la priorización de tecnologías emergentes de recobro térmico es compleja la asignación o confiabilidad de costos e ingresos, ya que en su mayoría se encuentran en casos implementados a escala de campo y en proyectos piloto (Bustos, S. M., \& Angarita, W. Y. 2013). Por ende, este estudio presenta una metodología que de forma rápida permite evaluar y preseleccionar campos petroleros que serían prospectos para implementar la tecnología Solar EOR, basado en criterios factibles de evaluar a la luz de su desarrollo técnico actual (Chilingar, G. V., \& Yen, T. F. 1984).

Entre los métodos de recuperación mejorada se encuentran: los métodos térmicos, los de inyección de gas y los métodos químicos. La aplicación óptima de cada método depende de las propiedades de la roca, de los fluidos y de la interacción roca-fluido; es decir, de la temperatura, la presión, la profundidad, la zona productiva neta, la permeabilidad, las saturaciones de petróleo residual y agua, la porosidad y las propiedades del fluido del yacimiento, tales como la gravedad API y la viscosidad. Para este caso en particular, se hace énfasis en los métodos térmicos de recuperación mejorada, estos se caracterizan porque incrementan el factor de recuperación de crudos y son altamente utilizados en crudos de naturaleza pesados y extra - pesados, están técnicas basadas en el principio de transferencia de energía térmica hacia el yacimiento, originando un aumento de movilidad del aceite como resultado de la reducción de su viscosidad y finalmente mejorar la fluidez hacia el productor; los métodos térmicos se pueden clasificar en: (1) combustión in situ, (2) inyección de agua caliente, (3) calentamiento térmico (eléctrico) y (4) inyección de vapor

Existen dos formas de suministrar calor a la formación. La primera de ellas es desde el exterior a través de inyección de vapor, inyección de agua caliente o suministro de energía eléctrica a través de pozo inyectores en las cercanías del peso productor. La segunda es generando el calor en el interior de la formación por combustión, en donde el aceite in situ funciona como combustible y se inyecta aire (o cualquier otro fluido que contenga oxígeno) para que haga la acción del oxidante (Márquez, 2016). En la Tabla 1 se enuncian las características básicas de cada uno de los procesos de recobro térmico.

\section{Metodología}

La definición de los factores determinantes para la implementación de SOLAR EOR en el país, pasa en primera instancia por la identificación de los parámetros esenciales para la aplicación de la técnica (Choudry, S. A. et al, 2018). Es importante clarificar que el objetivo fundamental de este trabajo es discretizar los puntos clave para la exitosa ejecución de proyectos de solar EOR para posteriormente seleccionar los campos candidatos para la implementación de esta técnica. El flujograma de trabajo se encuentra continuación:

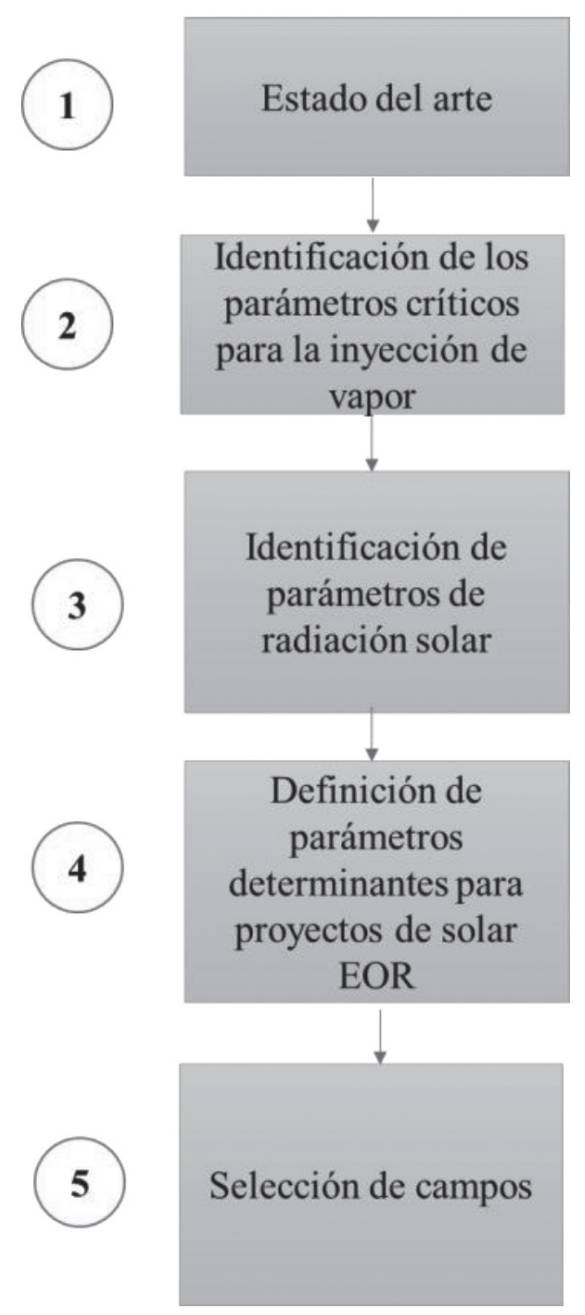

Figura 1. Flujo de trabajo para definición de parámetros determinantes para proyectos de solar EOR. 


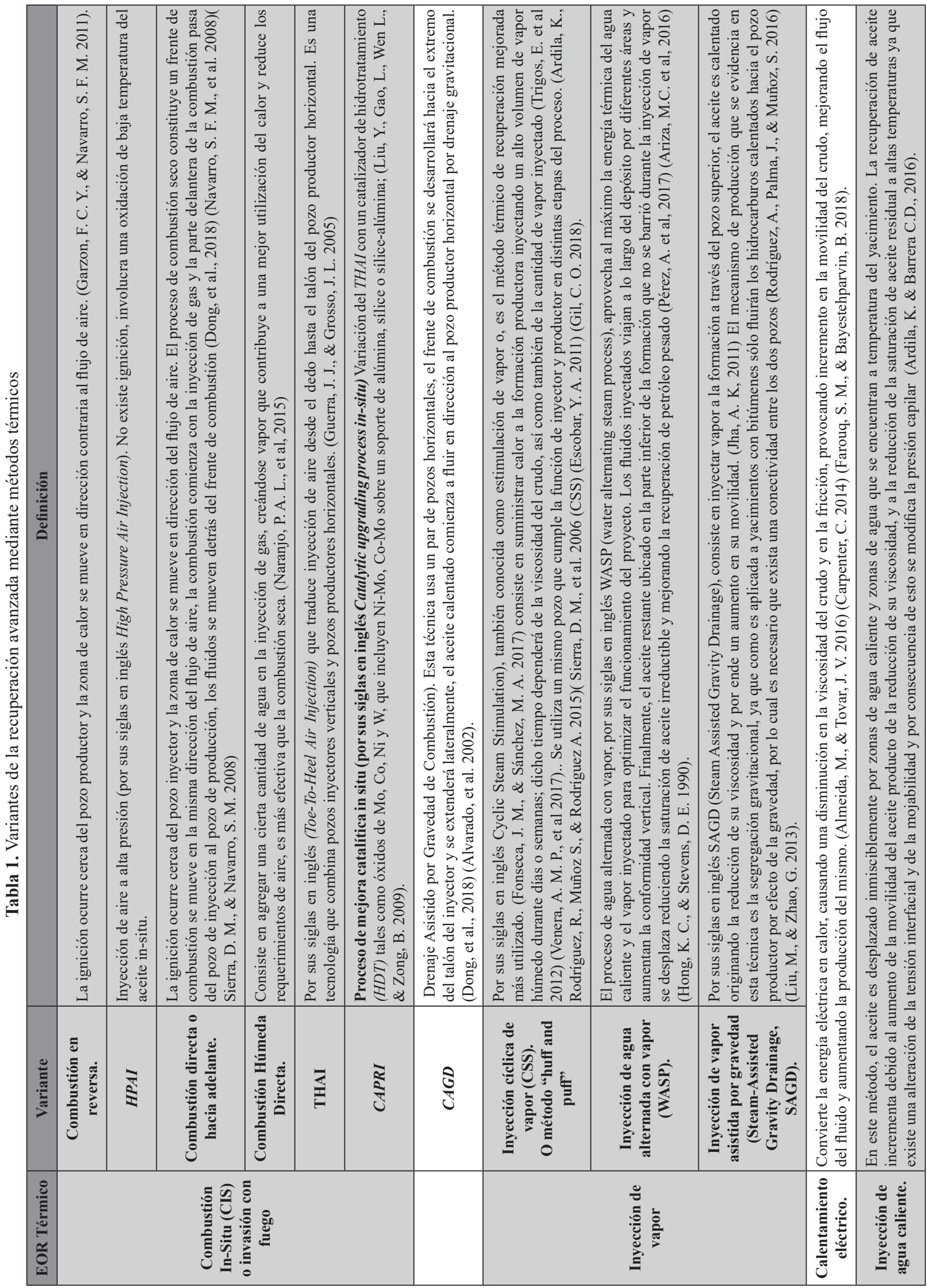


Inicialmente fue llevado a cabo un levantamiento bibliográfico sobre las principales técnicas de recuperación avanzada de petróleo a partir de procesos térmicos, posteriormente fueron comparadas las tecnologías de energía solar en cuanto a su viabilidad económica y eficiencia energética. En la Tabla 2 quedan evidenciados los parámetros que fueron objeto de comparación. Subsecuentemente, fueron detallados los factores clave que aseguran el éxito de los procesos de recobro térmico, tales como la generación de vapor en superficie que permite su eventual llegada al reservorio, teniendo en cuenta las pérdidas de vapor en la tubería de producción y las técnicas empleadas para evitar la pérdida de vapor generada por la falta de control del frente de inyección. Por otra parte, en cuanto a las tecnologías de energía solar, los parámetros determinantes pueden enumerarse: radiación solar, tecnología de concentración de energía solar (CSP) seleccionada, radiación incidente sobre la superficie terrestre, disponibilidad de las facilidades de superficie para la instalación del parque solar y señales de indicación de temperatura con la cual cuenta la tecnología. Acto seguido, fueron determinadas las características petrofísicas y de propiedades de los fluidos necesarios para que un yacimiento fuese candidato a trabajos de recuperación por métodos térmicos.

Ponderando cada uno de los factores que fueron expuestos encima, la implementación de esta tecnología en un campo petrolífero depende principalmente de los siguientes factores:

1. La tecnología de concentración de energía solar (CSP) seleccionada.

2. Radiación incidente sobre la superficie terrestre.

3. Irradiación normal directa (DNI).

4. Propiedades petrofísicas del yacimiento y los fluidos que se encuentran en él.

5. Mecanismo de recobro que se le han implementado al Campo.

\section{Resultados y discusiones}

Como fue planteado en la metodología, cada uno de los cinco parámetros críticos que fueron evaluados. (Figura 2)

La incidencia de la irradiación solar uno de los factores decisivos. Según especificaciones técnicas de algunos colectores solares comerciales un valor adecuado (Vogel, 2010) promedio de irradiación global horizontal para obtener resultados satisfactorios es de $4,2 \mathrm{KWh} / \mathrm{m}^{2} / \mathrm{dia}$.

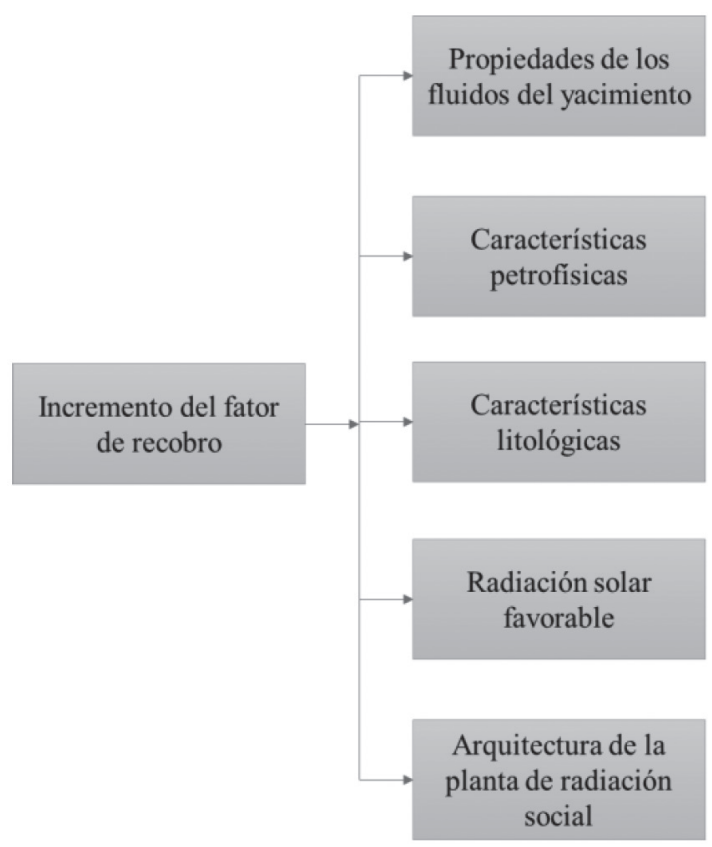

Figura 2. Parámetros para el incremento de factor de recobro.

La consulta arrojó que la irradiación normal directa (DNI) en Colombia, en Colombia, el IDEAM es la entidad oficial encargada, a nivel nacional, de hacer el seguimiento a la radiación y cuenta con la red más grande de estaciones y de mayor cobertura; los valores de irradiación global horizontal media recibida en superficie oscilan, desde un máximo de entre 5,5 y 6,0 $\mathrm{kWh} / \mathrm{m}^{2}$ por día (entre 2.000 y $2.200 \mathrm{kWh} / \mathrm{m}^{2}$ por año) en el norte de la región Caribe, hasta mínimos de entre 3,0 y $3,5 \mathrm{kWh} / \mathrm{m}^{2}$ por día (entre 1.100 y $1.280 \mathrm{kWh} /$ $\mathrm{m}^{2}$ por año), en sectores de la región Pacífica (Gupta et al. 2017).

Colombia, debido a su posición geográfica, es favorecida con una gran disponibilidad de recurso solar; como se puede observar en la Figura 3, las zonas de mayor intensidad de radiación solar global, superiores a los $4,5 \mathrm{kWh} / \mathrm{m}^{2}$ por día, son: las Islas de San Andrés y Providencia, amplios sectores de la región Caribe, Vichada, Arauca, Casanare, Meta, el norte, sur y oriente de Antioquia, el centro y norte de Boyacá, el occidente de Cundinamarca, el oriente y centro del Tolima, el norte del Huila; la zona que se inicia en el norte del Cauca, atraviesa el Valle del Cauca de sur a norte y llega hasta el Eje Cafetero, así como sectores puntuales del norte de Nariño, del norte de Norte de Santander y del suroriente de Santander. Los valores más altos (superiores a los $5,5 \mathrm{kWh} / \mathrm{m}^{2}$ por día) se muestran en pequeños sectores del centro y norte de La Guajira (Benavides, et al., 2017). 
Tabla 2. Comparación de Tecnologías Solares CSP

\begin{tabular}{|c|c|c|c|c|c|}
\hline Tecnología CSP & $\begin{array}{c}\text { Canal } \\
\text { parabólico }\end{array}$ & Torre solar & Lineal Fresnel & Canal cerrado & Plato Stirling \\
\hline $\begin{array}{l}\text { Madurez de la } \\
\text { tecnología }\end{array}$ & $\begin{array}{c}\text { Comercialmente } \\
\text { probado }\end{array}$ & $\begin{array}{l}\text { Proyectos comerciales } \\
\text { piloto }\end{array}$ & $\begin{array}{l}\text { Proyecto de } \\
\text { piloto }\end{array}$ & $\begin{array}{l}\text { Proyectos } \\
\text { comerciales piloto }\end{array}$ & Demostración \\
\hline $\begin{array}{l}\text { Riesgo de desarrollo } \\
\text { tecnológico }\end{array}$ & Bajo & Medio & Medio & Bajo & Medio \\
\hline $\begin{array}{c}\text { Temperatura de } \\
\text { funcionamiento }\left({ }^{\circ} \mathrm{C}\right)\end{array}$ & Hasta 550 & Hasta 565 & Hasta 550 & Hasta 350 & Hasta 750 \\
\hline Receptor / absorbedor & $\begin{array}{c}\text { Absorbente } \\
\text { unido al colector, } \\
\text { se mueve con } \\
\text { colector, diseño } \\
\text { complejo }\end{array}$ & $\begin{array}{l}\text { Superficie o cavidad } \\
\text { externa, receptor fijo }\end{array}$ & $\begin{array}{l}\text { Amortiguador } \\
\text { fijo, reflector } \\
\text { secundario }\end{array}$ & Tubo receptor fijo & $\begin{array}{l}\text { Absorbedor } \\
\text { unido al } \\
\text { colector, se } \\
\text { mueve con } \\
\text { colector }\end{array}$ \\
\hline Líquido de trabajo & $\begin{array}{c}\text { Aceite de } \\
\text { transferencia } \\
\text { de calor o sal } \\
\text { fundida }\end{array}$ & $\begin{array}{c}\text { Agua tratada, generación } \\
\text { directa de vapor o } \\
\text { fundido }\end{array}$ & $\begin{array}{c}\text { Agua tratada, } \\
\text { generación } \\
\text { directa de vapor }\end{array}$ & $\begin{array}{c}\text { Agua mínimamente } \\
\text { tratada, generación } \\
\text { directa de vapor }\end{array}$ & $\mathrm{n} / \mathrm{a}$ \\
\hline Solución de lavado & $\begin{array}{c}\text { Camiones } \\
\text { manuales y } \\
\text { lavado de manos }\end{array}$ & $\begin{array}{c}\text { Camiones manuales y } \\
\text { semiautomatizados }\end{array}$ & $\begin{array}{l}\text { Robots de } \\
\text { limpieza } \\
\text { manuales y } \\
\text { prototipo }\end{array}$ & $\begin{array}{l}\text { Robots de limpieza } \\
\text { automáticos } \\
\text { comprobados con } \\
\text { reciclaje de agua }\end{array}$ & $\begin{array}{l}\text { Manual, lavado } \\
\text { a mano }\end{array}$ \\
\hline $\begin{array}{c}\text { Uso de la tierra } \\
\text { (toneladas de vapor por } \\
\text { día por hectárea) }\end{array}$ & 6 & 12 & 24 & 33 & $\mathrm{n} / \mathrm{a}$ \\
\hline $\begin{array}{l}\text { Velocidad máxima del } \\
\text { viento de operación }\end{array}$ & Bajo & Bajo & Medio & Alto & Bajo \\
\hline $\begin{array}{l}\text { Capacidad total } \\
\text { Instalada (MW) }\end{array}$ & $>1500$ & $<50$ & $<50$ & $>1500$ & $\mathrm{n} / \mathrm{a}$ \\
\hline $\begin{array}{c}\text { Tamaño de capacidad } \\
\text { Generadora típica }(\mathrm{MW})\end{array}$ & $14-250$ & $50-370$ & $5-30$ & $>370$ & $10-25(\mathrm{~kW})$ \\
\hline $\begin{array}{c}\text { Porcentaje de eficiencia } \\
(\%)\end{array}$ & $14-16$ & $15-17$ & $8-13$ & $>30$ & $>30$ \\
\hline $\begin{array}{c}\text { Capacidad de } \\
\text { almacenamiento térmico } \\
(\mathrm{hr})\end{array}$ & $0.0-7.5$ & $1.0-15$ & 0.5 & $>15$ & $\mathrm{n} / \mathrm{a}$ \\
\hline $\begin{array}{l}\text { Tiempo de construcción } \\
\text { (años) }\end{array}$ & $1-3$ & $1-2$ & $1-2$ & $1-2$ & 1 \\
\hline Tiempo de vida (años) & $>30$ & 30 & $\mathrm{n} / \mathrm{a}$ & $>30$ & $>15$ \\
\hline
\end{tabular}

Fuente: Agencia Internacional de Energías Renovables (IRENA), Tecnologías de energía renovable: serie de análisis de costos.

La Tabla 3 destaca las diferencias en $D N I$ en Departamentos que cumplen con los criterios de $D N I$ para la implemetación de Solar EOR seleccionados de
Colombia. Los promedios anuales estimados en los Departamentos seleccionados oscilan entre 1.642 a $2.190 \mathrm{kWh} / \mathrm{m}^{2} /$ año. 
Tabla 3. DNI en Departamentos de interés para SOLAR EOR

\begin{tabular}{|c|c|c|}
\hline Departamento & Radiación diaria promedio $\left(\mathbf{k W h} / \mathbf{m}^{2} / \mathbf{d i ́}\right)$ & Radiación anual estimada $\left(\mathbf{k W h} / \mathbf{m}^{2} / \mathbf{a n ̃ o}\right)$ \\
\hline Boyacá & $4,5-5,0$ & $1.642-1.825$ \\
\hline Antioquia & $4,5-5,0$ & $1.642-1.825$ \\
\hline Meta & $4,5-5,0$ & $1.642-1.825$ \\
\hline Bolívar & $5,0-5,5$ & $1.825-2.007$ \\
\hline Tolima & $5,0-5,5$ & $1.825-2.007$ \\
\hline Casanare & $5,0-5,5$ & $1.825-2.007$ \\
\hline Arauca & $5,0-5,5$ & $1.825-2.007$ \\
\hline Vichada & $5,0-5,5$ & $1.825-2.007$ \\
\hline Guajira & $5,5-6,0$ & $2.007-2.190$ \\
\hline Cesar & $5,5-6,0$ & $2.007-2.190$ \\
\hline Magdalena & $5,0-6,0$ & $1.825-2.190$ \\
\hline Atlántico & $5,0-6,0$ & $1.825-2.190$ \\
\hline
\end{tabular}

Fuente: Benavides, et al. (2017).

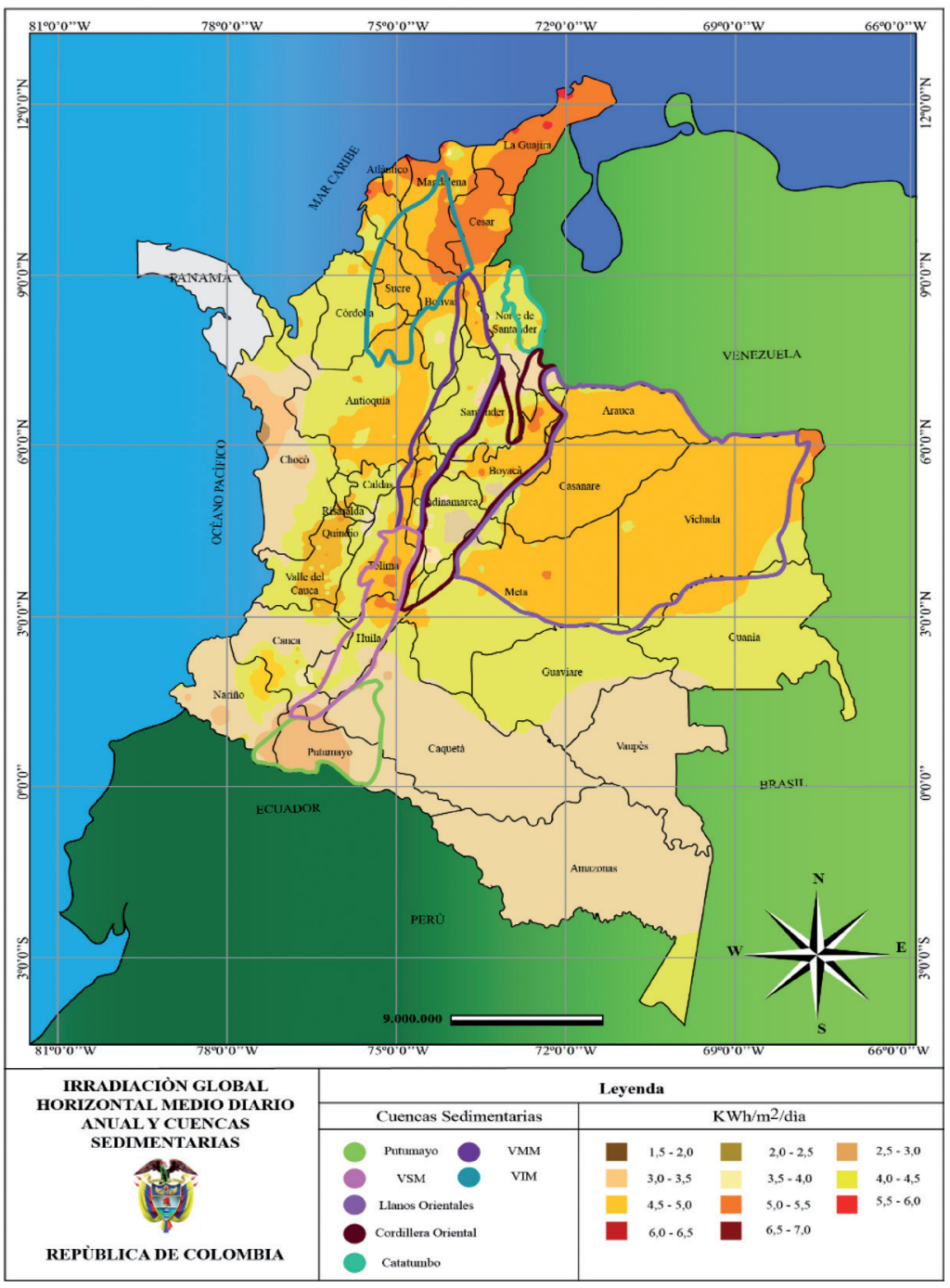

Figura 3. Irradiación Global Horizontal en Colombia.

Fuente: Modificado (Barrero, et al, 2007). 
La producción actual de petróleo en Colombia se basa principalmente en procesos térmicos de extracción conocidos como en una primera instancia como Procesos de Levantamiento Artificial y en una etapa posterior Recobro Mejorado (EOR/IOR). Ecopetrol, la principal empresa productora del país cuenta actualmente con cerca de 52 proyectos de Recobro No Térmico $(I O R)$ en 44 campos y cerca de 6 proyectos con Recobro Térmico (EOR). De acuerdo con los Departamentos seleccionados que cumplen con los requerimientos de $D N I$ para la implementación de la tecnología Solar EOR se procede a identificar los campos petrolíferos ubicados en estas zonas de interés que a su vez cumplen con los parámetros de yacimiento y de fluidos mencionados anteriormente en la Tabla 3; a continuación, en la Tabla 4 se encuentran los campos en mención. (Ecopetrol S.A. 2014)

La radiación solar y el tipo de mecanismo de producción que presenta el yacimiento es importante en la determinacion de la implementacion de Solar $E O R$, los campos ubicados que presentan recobro térmico son: en la Cuenca del Valle Medio del Magdalena se encuentra el campo Teca-Cocorná que presenta una gravedad $A P I$ de 12.5 , el mecanismo de producción es drenaje gravitacional y de compactacion de la formación, tiene implementado inyección cíclica de vapor e inyección continua de vapor. El campo Jazmín, el crudo es bastante viscoso con una gravedad API de 11.5, gas en solución con expansión térmica, segregación gravitacional y empuje por capa de gas, es una yacimiento somero que tiene implementado el método de recobro térmico inyección cíclica de vapor. El Campo Girasol presenta una gravedad API de 11.2, el método de recobro es inyección cíclica de vapor; finalmente el Campo Moriche presenta una gravedad $A P I$ de 13.4 actualmente su producción se favorece debido al método de recobro térmico inyección cíclica de vapor. En la cuenca sedimentaria de los Llanos Orientales, se encuentra el campo Quifa, presenta una gravedad $A P I$ de 13.73 , a este campo se le realizó un proyecto piloto de recobro térmico de combustión seca directa el cual no cumplió con los objetivos establecidos, el mecanismo de producción del yacimiento es empuje hidraulico debido a la presencia de un acuifero activo. El campo Quifa está ubicado en la Cuenca Sedimentaria que recibe más irradiación solar normal directa, debido al mecanismo de producción que es un acuífero activo no es factible realizar un recobro térmico, ya que perdería eficiencia.

Por otra parte, en cuanto a la tecnología más adecuada es recomendable usar un colector de tipo canal cerrado. Despliega una tecnología cilindro-parabólica avanzada. En este diseño de campo solar, los espejos, el sistema de puntería y otros componentes delicados están protegidos dentro de una estructura de invernadero (Glasspoint. 2018). Los espejos rastrean automáticamente el sol durante todo el día y concentran la luz solar en un tubo estacionario de la caldera que contiene agua. El calor del sol hierve el agua para producir vapor a alta presión para EOR (Gregory, et al., 2014). De igual manera, se establece la medición de temperatura a través del circuito mostrado en la Figura 4.

Así, la Tabla 4 resume los campos seleccionados como potenciales candidatos para implementación de solar EOR.

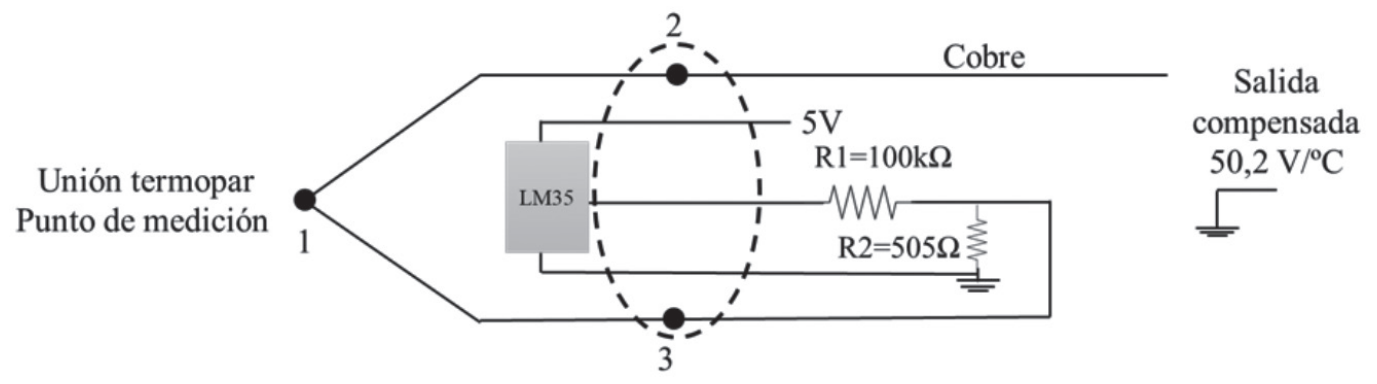

Figura 4. Medición de temperatura y medición de junta fria. Fuente: Modificado (Souza y Canto, 2004).

Los campos que se encuentran en la Cuenca del Valle Medio del Magdalena son prospectos para implemetar solar EOR ya que son campos que presentan pozos someros (profundidad menor a 4000 pies) y su gravedad $A P I$ es menor a $25^{\circ}$. El campo Moriche presenta un espesor de formación de 526 pies, el campo Jazmín 215 pies y una profundidad de 1200 a 2500 pies; el Campo Girasol posee un espesor de formación que varía entre 15-35 pies. 
De esta manera, el aprovechamiento de energía proveniente del sol para el calentamiento de agua, generación de vapor para su posterior inyección, es una posibilidad latente para el desarrollo sostenible de la industria de los hidrocarburos en Colombia.

Tabla 4. Campos petrolíferos seleccionados para la implementación de Solar EOR.

\begin{tabular}{|c|c|c|c|c|c|c|}
\hline Campo & Departamento & Cuenca & API & $\begin{array}{c}\text { Producción } \\
\text { (BPD) }\end{array}$ & $\begin{array}{c}\text { Método de recobro } \\
\text { utilizado }\end{array}$ & Detalles \\
\hline Teca - & Antioquia & $\begin{array}{c}\text { Valle Medio del } \\
\text { Magdalena }\end{array}$ & 12,5 & $1.264,54$ & $\begin{array}{c}\text { Inyección ciclica de } \\
\text { vapor e inyección } \\
\text { continua de vapor }\end{array}$ & $\begin{array}{c}\text { Se implementa } \\
\text { actualmete }\end{array}$ \\
\hline Nare & Antioquia & $\begin{array}{c}\text { Valle Medio del } \\
\text { Magdalena }\end{array}$ & 12,5 & 198,92 & $\begin{array}{c}\text { Inyección cíclica de } \\
\text { vapor }\end{array}$ & $\begin{array}{c}\text { Se implementa } \\
\text { actualmete }\end{array}$ \\
\hline Jazmín & Boyacá & $\begin{array}{c}\text { Valle Medio del } \\
\text { Magdalena }\end{array}$ & $11,5-12,5$ & $4.260,43$ & $\begin{array}{c}\text { Inyección cíclica de } \\
\text { vapor }\end{array}$ & $\begin{array}{c}\text { Se implementa } \\
\text { actualmete }\end{array}$ \\
\hline Moriche & Boyacá & $\begin{array}{c}\text { Valle Medio del } \\
\text { Magdalena }\end{array}$ & $12,4-15,8$ & $11.204,71$ & $\begin{array}{c}\text { Inyección cíclica de } \\
\text { vapor }\end{array}$ & $\begin{array}{c}\text { Se implementa } \\
\text { acutualmete }\end{array}$ \\
\hline Girasol & Boyacá & $\begin{array}{c}\text { Valle Medio del } \\
\text { Magdalena }\end{array}$ & 11,4 & $3.155,75$ & $\begin{array}{c}\text { Inyección cíclica de } \\
\text { vapor }\end{array}$ & $\begin{array}{c}\text { Se implementa } \\
\text { acutualmete }\end{array}$ \\
\hline Chichimene & Meta & Llanos orientales & 8.93 & $51.835,75$ & $\begin{array}{c}\text { Combustión in situ } \\
\text { acutualmete }\end{array}$ \\
\hline Quifa & Meta & Llanos Orientales & 13,9 & $42.407,93$ & $\begin{array}{c}\text { Se implementa } \\
\text { combustión seca directa } \\
\text { (Recobro Adicional } \\
\text { Térmico Sincronizado) }\end{array}$ & $\begin{array}{c}\text { No cumplió con la } \\
\text { meta, establecida }\end{array}$ \\
\hline
\end{tabular}

Fuente: Producción fiscalizada de petróleo por campo en superficie 2018, 2. Agencia Nacional de Hidrocarburos ANH, 2018.

\section{Conclusiones y recomendaciones}

- La tecnología Solar EOR se consolida como alternativa viable a la producción de vapor a gas para la industria petrolera debido a que puede generar la misma calidad de vapor que el gas natural, alcanzando temperaturas de hasta $750^{\circ} \mathrm{F}$ y 2,500 PSI.

- Una vez revisados los parámetros de yacimiento $y$ el mapeamento de Se concluye que los campos: Teca-Cocorna, Nare, Jazmín, Moriche, Girasol y Chichimene son campos potenciales para la aplicación de la tecnología expuesta. La implementación de Solar EOR podría reducir la demanda requerida de gas natural para $E O R$, que puede redirigirse a otras actividades económicas tales como generación de energía, desalinización de agua y como materia prima y energía para procesos industriales.

- Además de los beneficios económicos, la implementación de la tecnología SOLAR EOR trae consigo la posibilidad de estrechar la relación con la comunidad y los estados, lo anterior, dado a la reducción de emisiones indirectas. Comprometiendo de esta manera a la industria del petróleo y gás con la agenda de sustentabilidad de la ONU.
- Se recomienda calcular la cantidad de $\mathrm{CO}_{2}$ emitidas mediante la construcción y posterior puesta en marcha del proyecto. De igual manera, en caso de que la tecnología planteada reduzca las emisiones de $\mathrm{CO}_{2}$ es necesario cuantificar su costo de abatimiento.

- Se recomienda realizar los trabajos de ingeniería básica, conceptual y de diseño para los campos candidatos.

\section{Referencias}

1. Agencia Nacional de Hidrocarburos. (2018). Producción fiscalizada de petróleo por campo en superficie (barriles promedio por día calendario - bpdc). Bogotá, Colombia.: ANH. Recuperado de http://www.anh.gov.co/

2. Almeida, M., \& Tovar, J. V. (2016). Evaluación de los mecanismos de recuperación térmica para aumentar el factor de recobro del crudo pesado y extrapesado en la faja petrolífera del Orinoco "Hugo Chavez Frias" (tesis de pregrado). Universidad Nacional Experimental Politécnica de la Fuerza Armada Nacional Bolivariana, San Tomé, Venezuela. 
3. Alvarado, D., Bánzer S. C., \& Rincón, A. (Ed.). (2002). Recuperación Térmica de Petróleo, Caracas, Venezuela.

4. Ardila, C. A., \& Barrera, C. D. (2016). Evaluación técnico - financiera de compatibilidad de secuestrantes de H2S en fluidos de perforación y completamiento en operaciones del campo jazmín mediante pruebas de laboratorio (Trabajo de grado). Fundación Universidad de América, Bogotá, Colombia.

5. Ardila, K., Rodríguez, R., Muñoz S., \& Rodríguez A. (2015). Uso de un solvente como alternativa para mejorar la inyección cíclica de vapor en un yacimiento de crudo pesado móvil. Revista fuentes: el Reventón energético, 13(1), 33-45.

6. Barrero, D., Pardo, A., Vargas, C., \& Martínez J. (2007). Colombian Sedimentary Basins: Nomenclature, Boundaries and Petroleum Geology, a New Proposal. Bogotá, Colombia.: ANH. Recuperado de http://www.anh.gov.co/ Informacion-Geologica-y-Geofisica/Cuencassedimentarias/Documents/colombian sedimentary_basins.pdf

7. Benavides, H. O., Simbaqueva, O., \& Zapata, H. J. (2017). Atlas de radiación solar, ultravioleta y ozono de Colombia. Bogotá D.C., Colombia.: Atlas IDEAM. Recuperado de http://atlas. ideam.gov.co/visorAtlasRadiacion.html

8. Bustos, S. M., \& Angarita, W. Y. (2013). Aplicación de la tecnología visbreaking para el mejoramiento del crudo del campo Jazmín (trabajo de grado). Universidad Industrial de Santander, Bucaramanga, Colombia.

9. Carpenter, C. (2014). Downhole Electrical Heating for Enhanced Heavy-Oil Recovery. Journal of Petroleum Technology, 66(03), 132-134. doi: 10.2118/0314-0132-JPT.

10. Chilingar, G. V., \& Yen, T. F. (1984). Enhanced Recovery of Residual and Heavy Oils (Second Edition), edited by M. M. Schumacher, Noyes Data Corporation, Park Ridge, NJ, 1980. 389. Energy Sources, 7(4), 403-418. doi: 10.1080/00908318408908095.

11. Choudry, S. A., Müller, S., Alber, U., Riedel, F., \& Landgrebe, D. (2018). A Multidimensional Assessment and Selection Methodology: Optimized Decision-making of Joining Technologies in Automobile Body Development. Elsevier Procedia Manufacturing, 21, 281-288. doi: 10.1016/j. promfg.2018.02.122.

12. Dong, X., Liu, H., Wu, K., \& Chen, Z. (2018). EOR potential in the post steam injection era: Current and future trends. SPE Improved Oil Recovery Conference. doi: 10.2118/190195-MS.

13. Escobar, Y. A. (2011). Evaluación del completamiento de subsuelo para la inyección de vapor y el sistema de levantamiento artificial en los pozos del campo Jazmín orientada a la reducción del lifting cost (tesis de especialización). Universidad Industrial de Santander, Bucaramanga, Colombia.

14. Farouq, S. M., \& Bayestehparvin, B. (2018). Electrical Heating - Doing the Same Thing Over and Over Again .... SPE Canada Heavy Oil Technical Conference. doi: 10.2118/189724-MS.

15. Fonseca, J. M., \& Sánchez, M. A. (2017). Evaluación de la inyección localizada de vapor con la técnica huff and puff para ocho pozos en el campo Teca-Cocorná mediante simulación numérica (trabajo de grado). Fundación Universidad de América, Bogotá, Colombia.

16. Garzon, F. C. Y., \& Navarro, S. F. M. (2011). Una nueva mirada a la combustión in-situ: Tratando de romper un viejo paradigma. Revista Fuentes, 9(1).

17. Gil, C. O. (2018). Análisis técnico para determinar las fallas de las bombas de subsuelo del sistema de bombeo mecánico ocasionado por la inyección cíclica de vapor en el campo Moriche de Mansarovar Energy LTDA (trabajo de grado). Universidad Industrial de Santander, Bucaramanga, Colombia.

18. Guerra, J. J., \& Grosso, J. L. (2005). Segregated InSitu Combustion Process (SISC) y Toe to Heel Air Injection (THAI), alternativas potenciales de recobro mejorado en campos Colombianos de crudo medio y pesado. Paper ACIPET, Articulo elegido por el Comité Técnico para presentación en el XI Congreso Colombiano de Petróleo y gas, organizado por ACIPET. Bogotá D.C, Colombia.

19. Gupta, S., Guédez, R., \& Laumert, B. (2017). Market potential of solar thermal enhanced oil recovery-a techno-economic model for Issaran oil field in Egypt. AIP Conference Proceedings, 1850(1)1850, 190001. doi:10.1063/1.4984573.

20. IRENA, R. E. S. (2015). International Renewable Energy Agency. Renewable Energy Target Setting, Abu Dhabi, UAE.

21. Jha, A. K, Joshi, N., \& Singh, A. (2011). Applicability and assessment of microwave assisted gravity drainage (MWAGD) applications in Mehsana heavy oil field, India. SPE Heavy Oil Conference and Exhibition. doi: 10.2118/149591-MS 
22. Liu, M., \& Zhao, G. (2013). A performance comparison study of electromagnetic heating and SAGD process. SPE Heavy Oil Conference - Canada, 3, 2119-2129. doi: 10.2118/165547-MS.

23. Liu, Y., Gao, L., Wen L., \& Zong, B. (2009). Recent advances in heavy oil hydroprocessing technologies. Recent Patents on Chemical Engineering, 2(1), 22-36. doi: 10.2174/2211334710902010022.

24. Márquez, D. S. (2016). Recuperación mejorada mediante el uso de energía geotérmica (tesis de pregrado). Universidad Autónoma de México, Ciudad de México, México.

25. Naranjo, P. A. L., Correa, D. L. B., Navarro, S. F. M., \& Rodriguez, A. O. (2015). Inyección de vapor en medianos. recuperación y rentabilidad. Revista Fuentes, 13(1), 21-31.

26. Navarro, S. F. M., Olivares, C. A. P., \& Becerra, E. M. T. (2008). Procesos de desplazamiento miscibles y su incidencia en el comportamiento de la inyección de vapor. Revista Fuentes, 6(2).

27. Pardo, D. F. (2015). Implicaciones de un Sector Petrolero inactivo. CAMPETROL Cámara Colombiana de bienes y servicios petroleros. Recuperado de http://campetrol. org/wp-content/uploads/noticias/boletin/ ESTUDIO-01_Implicaciones-sector-petroleroinactivo.pdf.

28. Rodríguez, A., Palma, J., \& Muñoz, S. (2016). Drenaje gravitacional asistido Con vapor, SAGD, aplicado a yacimientos de crudos pesados. Revista Fuentes: El Reventón Energético, 14(1), 35-42. doi: https://doi. org/10.18273/revfue.v14n1-2016003.

29. Schlumberger. (2018). Teor. Schlumberger. Recuperado de: https://www.glossary.oilfield. slb.com/es/Terms/t/teor.aspx.

30. Sierra, D. M., \& Navarro, S. M. (2008). modelo analítico para inyección continua de vapor en yacimientos estratificados de crudo pesado. Revista Fuentes, 6(1).

31. Sierra, D. M., Navarro, S. F. M., \& Tavera, C. P. S. (2006). Simulación de un piloto de inyección continua de vapor usando pozos horizontales. Fuentes: El reventón energético, 4(2), 2.

32. Souza, T. M. D., \& Canto, M. A. R. D. (2004). Sistema de monitoramento de fontes de energias renováveis. Procedings of the 5th Encontro de Energia no Meio Rural.

33. Venera, A. M. P., Tarazona, M. C. A., Castelblanco, A. X. R., \& Martínez, S. F. M. (2017). Técnicas para el mejoramiento de la inyección continua de vapor. Revista Fuentes, 15(1), 109-117.

34. Vidal, E. A. \& Fontalvo, C. A. (2018). Alternativa para la generación de gas natural sintético a partir de una fuente de energía renovable mediante tecnología "Power to Gas" en Colombia. Revista Fuentes: El reventón energético, 16(1), 71-79. doi: 10.18273/revfue. v16n1-2018006.

35. Vogel, W., \& Kalb, H. (2010). Large-scale solar thermal power: technologies, costs and development. John Wiley \& Sons

Recepción: 11 de julio de 2019

Aceptación: 02 de diciembre de 2019 